\title{
The reliability of the ICD-AIS map in identifying serious road traffic injuries from the Helsinki Trauma Registry
}

\author{
Noora K. Airaksinen ${ }^{\mathrm{a}, *}$, Mikko T. Heinänen ${ }^{\mathrm{b}, \mathrm{c}}$, Lauri E. Handolin ${ }^{\mathrm{b}}$ \\ a Kuopio Musculoskeletal Research Unit (KMRU), University of Eastern Finland, P.O. Box 1627, FI-70211, Kuopio, Finland \\ ${ }^{\mathrm{b}}$ Trauma Unit, Helsinki University Hospital, Topeliuksenkatu 5, PB 266, FI-00029, HUS, Helsinki, Finland \\ ${ }^{\mathrm{c}}$ Department of Orthopedics and Traumatology, University of Helsinki and Helsinki University Hospital, Finland
}

\section{A R T I C L E I N F O}

\section{Keywords:}

Traffic injuries

Injury severity

AIS

Injury coding

Mapping

ICD

\begin{abstract}
A B S T R A C T
Objective: The EU has recommended that its member countries compile statistics on the number of serious road traffic injuries. In Finland, the number of seriously injured road traffic patients is assessed using the International Classification of Diseases, $10^{\text {th }}$ Revision (ICD-10) and the automatic conversion tool (ICD-AIS map) developed by The Association for the Advancement of Automotive Medicine (AAAM). The aim of this study was to assess how reliably the ICD-AIS map identifies both serious injuries and seriously injured patients due to road traffic accidents.

Methods: Data was derived from the Helsinki Trauma Registry (HTR) and included 215 severe (New Injury Severity Score >15) trauma patients injured in road traffic accidents from the years 2016 and 2017. The severity ratings of injuries (Abbreviated Injury Scale, AIS 3+) and patients (Maximum Abbreviated Injury Scale, MAIS 3+) were determined by direct AIS coding of the HTR and were also generated by the ICD-AIS map based on ICD-10 injury codes. These two ratings were compared by injury mechanism and Injury Severity Score (ISS) body regions. The strength of agreement was described using Cohen's $\kappa$. The most common injury codes with errors in severity rating by the ICD-AIS map were presented.

Results: The number of seriously injured patients by the ICD-AIS map was $21 \%$ lower, and the number of serious injuries was $36 \%$ lower than the corresponding numbers by direct coding. The exact agreement of the injury ratings was $72 \%(\kappa=0.44,95 \% \mathrm{CI} 0.42-0.46)$. Most of the conversion errors were due to the simplicity of the ICD-10 codes used in Finland compared to those used in the ICD-AIS map (ICD-10-CM) and the missing codes from the ICD-AIS map. The most frequent misclassifications were due to multiple rib fractures, visceral organ injuries, some open fractures of extremities, and specific head injuries. Missing codes were most common in face, chest, and limb injuries.

Conclusions: The ICD-10 injury codes presently used in Finland should be more specific to permit reliable conversion results by the ICD-AIS map. The problem with missing codes should be considered more closely. When implementing the ICD-11, all detailed injury codes should be introduced.
\end{abstract}

(C) 2019 Elsevier Ltd. All rights reserved.

\section{Introduction}

To monitor and reduce serious road traffic injuries, the EU has recommended that its member countries compile statistics on the number of serious road traffic injuries according to a common definition known as MAIS 3+ [1]. MAIS 3+ is based on the Abbreviated Injury Scale (AISC) [2], which is a widely accepted injury severity rating method developed by the Association for the Advancement of Automotive Medicine (AAAM). The ideal use of AIS classification is to conduct the injury severity rating by a trained expert on the basis of the full medical records of a patient.

\footnotetext{
* Corresponding author at: Pahkakuja 5 B 8, FI-70150, Kuopio, Finland. E-mail address: nooraai@uef.fi (N.K. Airaksinen).
}

However, most hospitals are required to use the International Classification of Diseases (ICD) codes of injuries and the use of AIS requires extra work and special training. Therefore, AIS is not commonly used in clinical practice. Hence, the AAAM has developed an ICD-AIS mapping tool that generates the AIS classification from the ICD-9-Clinical modification (CM) or the ICD-10-CM injury code as an alternative to direct AIS coding by an expert [3,4]. Zonfrillo et al. [3] evaluated the ICD-AIS map and concluded that it seems to be useful in large databases when direct AIS coding is not available. However, it should not replace the use of a direct AIS coding by a trained expert in trauma registries.

Some countries, such as Finland, have followed the EU recommendation and started to compile statistics on serious road traffic injuries. According to the EU Horizon 2020 Safety Cube project survey [5], almost half of the respondent countries 
reported that they use the ICD-AIS map provided by AAAM for generating the number of seriously injured patients. While three EU countries (France, Germany, and Switzerland) reported use of direct AIS coding instead of ICD conversion, the content and coverage of hospital data vary [5]. In many other countries, also in Finland, the number of seriously injured patients is assessed using the ICD-AIS map, which is based on ICD-10 injury codes. In Finland, the data comes from the Finnish Hospital Discharge Registry (FHDR). Furthermore, in Finland direct AIS coding is used by the trauma registry of the Helsinki Trauma Registry (HTR), by regional traffic accident investigation teams, and occasionally by single researchers.

In addition to the conversion tool of AAAM, there are several other tools for generating ICD injury codes to AIS [6]. Some of them also generate Injury Severity Score (ISS) codes [7] and New Injury Severity Score (NISS) codes [8], which are AIS derivatives. Studies on the reliability of different tools are scarce because they require data that include direct AIS coding as a reference, which is rarely available. Furthermore, the conversion tools use various ICD and AIS versions and the quality of the input data varies, thus making the comparison of different studies quite difficult. In Finland, there is one previous study concerning the ICD-AIS map of AAAM [9]. In that study, Airaksinen et al. [9] concluded that the ICD-AIS map underestimates the number of seriously injured patients, and further research is needed especially with data that include more seriously injured patients.

The aim of this study was to assess how reliably the ICD-AIS map identifies both serious injuries and seriously injured patients due to road traffic accidents in the HTR.

\section{Methods}

The HTR (previously TR-THEL) is the trauma registry of the Helsinki University Hospital Trauma Unit and was established in 2006. It includes all NISS $>15$ trauma patients admitted to the resuscitation bay within $24 \mathrm{~h}$ after the accident; the HTR is thus a registry of severe trauma patients. Currently the HTR uses NISS rather than ISS (which was used until 2011) as inclusion criteria because NISS is superior to ISS in predicting mortality after blunt trauma [10]. The Helsinki University Hospital is a tertiary trauma centre with a catchment area of approximately 1.8 million. Five specially trained and direct AIS-coding certified trauma coordinators review the patient files and code the data using specialised software within 3 months of admission.

The collected data includes parameters for predictive models (patient and injury severity variables including AIS and ICD-10), process mapping (variables describing care provided), and resource utilization (variables describing the hospital resources needed). The validity of the data (completeness of cases and data and data accuracy) has been found to be excellent [11,12].

In the AIS classification, the human body is divided into nine body regions. Each injury description is assigned a six-digit unique numerical identifier, namely the "pre-dot code" that contains specific information of the injury. Furthermore, the seventh digit, the "post-dot code", is the AIS-severity code and is assessed on a scale from 1 to 6 (Table 1). Each injury is assessed separately and an individual patient may be assigned several AIS codes, one for each injury. Maximum AIS (MAIS) is defined as the patient's highest AIS severity code if there is more than one injury; MAIS depicts the overall severity of injuries [2]. Furthermore, the severity rating of injuries was defined as AIS 3+ (serious injury, including AIS 3-6 codes) or AIS 1-2 (minor injury) and the injured patient was defined as MAIS 3+ (seriously injured) or MAIS 1-2 (slightly injured).

Our data included HTR trauma patients from the years 2016 and 2017 with following road traffic accidents: pedestrian, bicycle,
Table 1

AIS body regions and example of full AIS code [2].

\begin{tabular}{ll}
\hline AIS body region \\
\hline 1 & Head (i.e. full AIS code 140678.2 Cerebrum, intraventricular haemorrhage) \\
2 & Face \\
3 & Neck \\
4 & Thorax \\
5 & Abdomen and pelvic contents \\
6 & Spine \\
7 & Upper extremity \\
8 & Pelvic and buttocks \\
9 & External (skin), thermal injuries and other trauma \\
\hline
\end{tabular}

motorcycle, and other motor vehicle accidents. The variables used in this study were all ICD-10 injury codes and AIS codes of the injuries, injury mechanism, and mortality. Furthermore, on the basis of AIS coding, the severity rating of injuries (AIS 3+ or not) and of patients (MAIS $3+$ or not) and the ISS body region of injuries were defined as new variables. The six ISS body regions do not match exactly with the nine AIS body regions presented previously. Hence, the ISS body regions of injuries were derived from the full AIS codes (Table 2). ISS body regions were chosen as they are commonly used in the international literature.

In addition to direct AIS coding, the severity rating of injuries was generated by the ICD-AIS map [13] from the ICD-10-CM to the AIS 2005/2008. The map solely generates the information on whether the injury code is serious (AIS $3+$ ) or not. Furthermore, the severity rating of patients (MAIS $3+$ or not) was also determined based on the injury severity rating by ICD-AIS map. The patients were assessed as seriously injured if the mapping tool correctly converted even one of the serious injury codes. Rating results were considered both by injury and by patient. Fatalities were excluded from patient-specific reviews, as the main purpose of the ICD-AIS map is the identification of seriously injured patients.

The severity ratings of the direct AIS coding and ICD-AIS map were compared with the ISS body regions. In addition, the severity ratings of patients (MAIS $3+$ ) were compared with the injury mechanism. The strength of agreement of injury ratings (AIS $3+$ or not) was described using Cohen's $\kappa$. Analysis was performed using SPSS software version 25 .

\section{Results}

The number of patients according to our criteria in the HTR was 215. Most patients were men (72.6\%) and the mean age of all patients was 44.3 years. The most common injury mechanism was a motor vehicle accident (40.0\%). Patients had a total of 1802 injuries; the average was 8.4 injuries per patient. All patients were seriously injured by the MAIS 3+ criteria (i.e. had at least one AIS 3+ injury). However, $35.0 \%$ of all injuries were serious (AIS 3+). Patient characteristics are described in detail in Table 3.

The total number of serious injuries (AIS 3+) was 630 by direct AIS coding and 405 by ICD-AIS map (Fig. 1 ). Thus, the ICD-AIS map underestimated $36 \%$ of serious injuries. Furthermore, the number of seriously injured patients (MAIS 3+), excluding 17 fatalities, was 198 by direct AIS coding and 157 by ICD-AIS map (Fig. 2), resulting in an underestimation of 21\%. The proportion of correct classification of MAIS 3+ patients by ICD-AIS map was highest in pedestrian accidents (86\%) and lowest in motorcycle accidents (76\%).

The exact agreement and the strength of agreement $(\kappa)$ in severity rating of injuries (AIS 3+ or not) between direct AIS-coding and ICD-AIS map are shown in Table 4 . Among all injuries, the exact agreement was $72 \%$ ( $\kappa=0.44,95 \%$ CI $0.42-0.46)$. The highest concordance between direct AIS coding and ICD-AIS map was in 
Table 2

Deriving the ISS body region from AIS code.

\begin{tabular}{|c|c|c|}
\hline \multicolumn{2}{|c|}{ ISS body region } & \multirow{2}{*}{$\begin{array}{l}\text { Corresponding AIS body region or } \\
\text { AIS code (the first digit) } \\
1 \text { or } 3 \text { alone and } 6 \text { if the } 4^{\text {th }} \text { digit } \\
\text { of the AIS code is } 2 \text { ( } 6 \times x \times 2 \times x . x)\end{array}$} \\
\hline 1 & Head and Neck & \\
\hline 2 & Face & 2 \\
\hline 3 & Chest & $\begin{array}{l}4 \text { alone and } 6 \text { if the } 4^{\text {th }} \text { digit of } \\
\text { the AIS code is } 4(6 \times x 4 \times x . x)\end{array}$ \\
\hline 4 & $\begin{array}{l}\text { Abdominal or pelvic } \\
\text { contents }\end{array}$ & $\begin{array}{l}5 \text { alone and } 6 \text { if the } 4^{\text {th }} \text { digit of } \\
\text { the AIS code is } 6 \text { ( } 6 \times x \times 6 \times x)\end{array}$ \\
\hline 5 & $\begin{array}{l}\text { Extremities or pelvic } \\
\text { girdle }\end{array}$ & 7,8 \\
\hline 6 & $\begin{array}{l}\text { External (skin) and thermal } \\
\text { injuries }\end{array}$ & 9 \\
\hline
\end{tabular}

Table 3

Characteristics of 215 patients and injuries in HTR.

\begin{tabular}{ll}
\hline Characteristic & $\mathrm{n}(\%)$ \\
\hline Male & $156(72.6)$ \\
Mean age, years (SD) & $44.3(20.4)$ \\
Mechanism of injury & \\
Pedestrian & $26(12.1)$ \\
Bicycle & $34(15.8)$ \\
Motorcycle & $69(32.1)$ \\
Motor vehicle & $86(40.0)$ \\
MAIS 3+ patients & $215(100)$ \\
Number of injuries & $1802(100)$ \\
AIS 3+ injuries & $630(35.0)$ \\
Fatalities & $17(7.9)$ \\
\hline
\end{tabular}

the injuries of extremities (82\%) and lowest in external injuries (24\%) (Table 4). However, according to direct AIS coding, there were no serious injuries among external injuries and the total number of external injuries was low $(n=21)$.

Detailed information on rating results according to ISS body regions is shown in Table 5. Conversion errors were most commonly underclassifications; the ICD-AIS map determined serious injuries as minor. Moreover, slightly over $10 \%$ of all injury codes were not found in the ICD-AIS map. Other errors were more infrequent.

Detailed information on misclassifications, missing codes, and undetermined codes is shown in Table 6A. Specific information about the severity ratings of the most common incorrectly converted injury codes is presented in Table 6B. Misclassifications were most frequently found for fractures of the ribs, sternum and thoracic spine (ICD-10 diagnoses S22.X; altogether 101 errors), trauma to thoracic organs (such as pneumothorax, haemothorax, and lung injuries [S27.X]; altogether 35 errors), abdominal organ injuries (such as liver, spleen, and bowel injuries [S36.X]; altogether 32 errors), and intracranial injuries (S06.X; altogether 27 errors).

\section{Discussion}

In this data, the number of seriously injured patients (MAIS 3+) was $21 \%$ lower and the number of serious injuries (AIS $3+$ ) was $36 \%$ lower by ICD-AIS map than by direct coding. When considering the injury rating result overall, the ICD-AIS map classified $72 \%$ of the ICD injury codes correctly. Conversion errors were most commonly underclassifications. The ICD-AIS map determined serious injuries as minor in $12 \%$ of the injury codes. Reverse classifications (minor to serious) were clearly less common. Another notable challenge with conversion was the missing codes in the ICD-AIS map; more than every tenth of all injury codes were not found in the map. Other conversion errors were more infrequent.

\section{Head and neck injuries}

Among head and neck injuries, misclassifications by the ICDAIS map occurred in several ICD-10 injury codes. However, the most common misclassified codes were S06.3 "Focal traumatic brain injury", S06.6 "Traumatic subarachnoid haemorrhage", and S02.0 "Fracture of vault of skull". Injury code S06.3 and all its subcodes are determined as serious in the ICD-AIS map. In Finland, there is only one code in use (S06.3), and most of these cases were serious by direct AIS-coding. Hence, several severity levels also appeared among S06.6-coded injures, resulting in misclassification by the ICD-AIS map. Injury code S06.6 is classified as minor injury by the ICD-AIS map but the duration of unconsciousness and the impact on severity is determined in its subcodes (ICD-10-CM). In Finland, however, there is only one code S06.6 in use, and in our data, according to direct AIS coding, over one fifth of these injuries were serious based on the duration of unconsciousness. These cases led to errors in the classification by the ICD-AIS map. Similarly, the ICD-AIS map assesses the code S02.0 as minor,

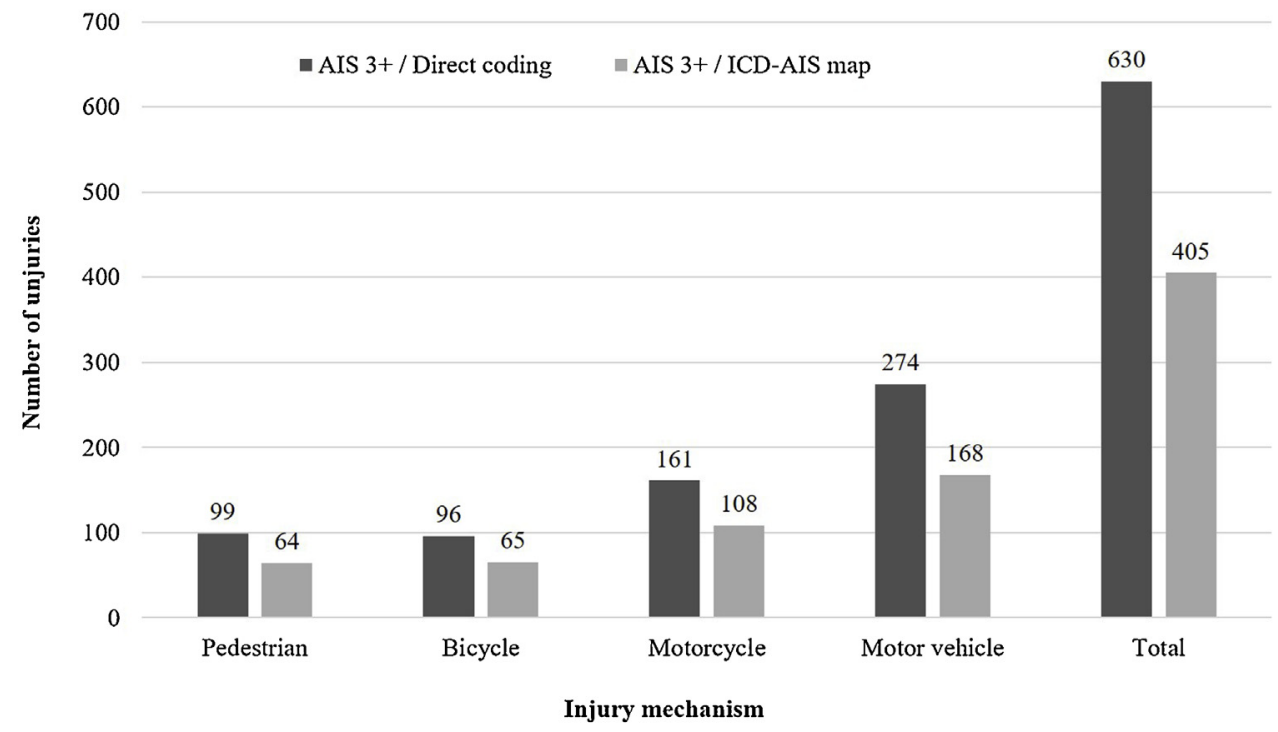

Fig. 1. Number of AIS 3+ injuries by injury mechanism according to direct AIS coding in HTR and ICD-AIS map. 


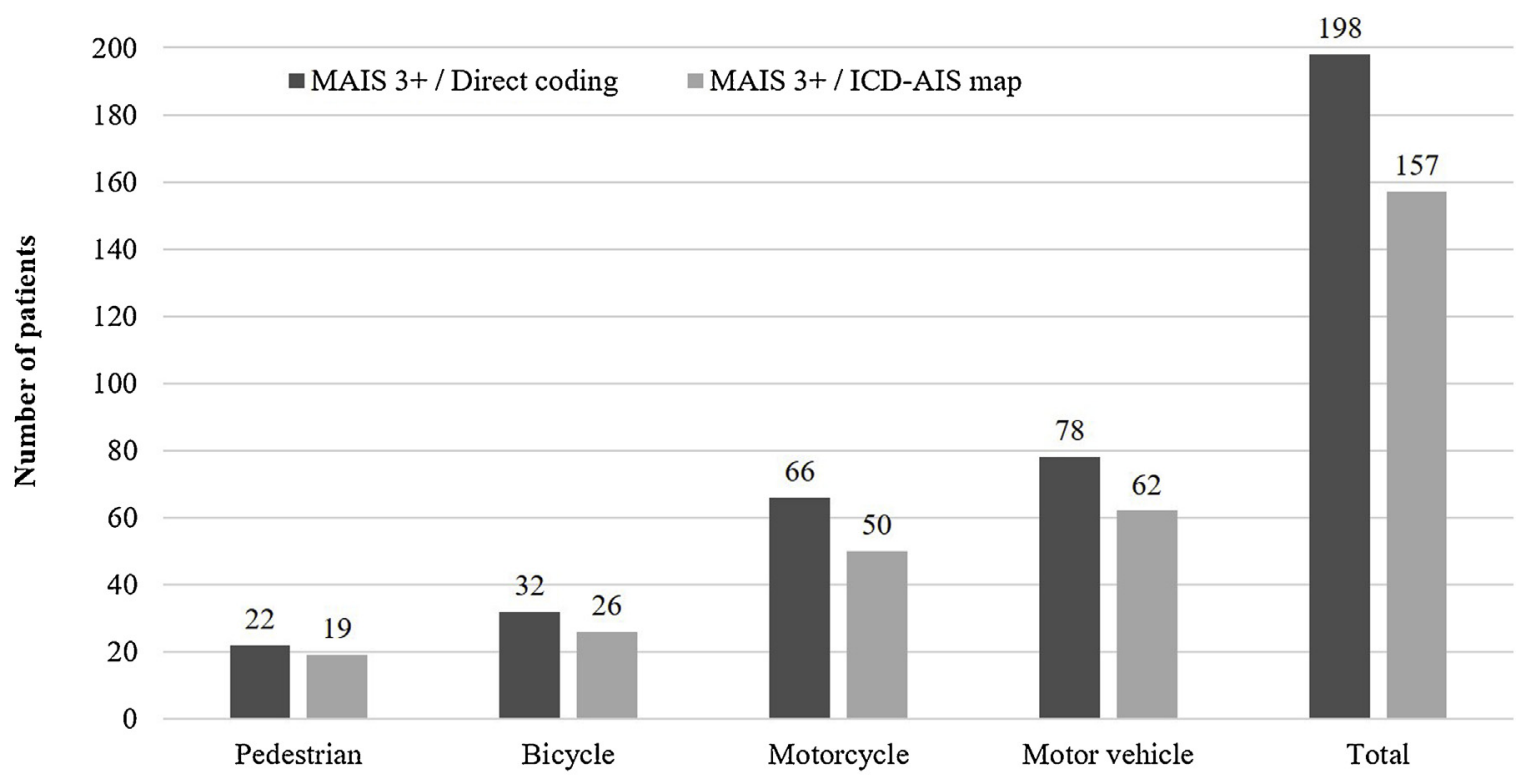

Injury mechanism

Fig. 2. Number of MAIS 3+ patients (excluding fatalities) by injury mechanism according to direct AIS coding in HTR and ICD-AIS map.

Table 4

Exact agreement and the strength of agreement $(\kappa)$ of severity ratings of injuries (AIS $3+$ or not) between direct coding and ICD-AIS map.

\begin{tabular}{lll}
\hline ISS body region of injury & Exact agreement & $\kappa(95 \% \mathrm{CI})$ \\
\hline Head and neck & $75 \%$ & $0.55(0.51-0.59)$ \\
Face & $76 \%$ & $0.11(0.07-0.15)$ \\
Chest & $63 \%$ & $0.32(0.29-0.36)$ \\
Abdominal or pelvic content & $66 \%$ & $0.10(0.05-0.15)$ \\
Extremities & $82 \%$ & $0.50(0.47-0.54)$ \\
External & $24 \%$ & - \\
All injuries & $72 \%$ & $0.44(0.42-0.46)$ \\
\hline
\end{tabular}

whereas open fracture of vault of skull is assessed as serious by subcode (ICD-10-CM). This code is not in use in Finland. In our data, well over half of the S02.0 injuries were serious by direct AIS coding and were misclassified by the ICD-AIS map.

The most common missing codes among head and neck injuries were S0X.7 and S1X.7, which are the codes for different kinds of multiple injuries. Furthermore, the most common undetermined injury codes were S0X.8 or S1X.8 (other injuries of head and neck) and S0X.9 or S1X.9 (undetermined injuries of different parts of head and neck).

\section{Facial injuries}

Missing codes were the main challenge in the severity rating of facial injuries by the ICD-AIS map. Most of the missing injury codes were minor by direct AIS coding. Therefore, considering the problem from the aspect of identifying serious injuries, the number of missing codes is not high. Misclassifications by the ICDAIS map were not common among facial injuries.

\section{Chest injuries}

The ICD-AIS map classified many serious chest injuries as minor, especially ICD-10 diagnosis code S22.4 "Multiple fractures of ribs". This injury is classified as serious according to AIS 2005/ 2008 if there are more than two fractured ribs. However, the ICD10 code does not separate the number of fractured ribs, nor does the ICD-AIS map. Thus, the code S22.4 was systematically rated as minor by the ICD-AIS map while majority of those cases were serious by direct AIS-coding.

It is noteworthy that the ICD-10 injury code S27.3 "Other and unspecified injuries of lung" and all its subcodes (ICD-10-CM) are determined as serious by the ICD-AIS map. However, in our data, almost one quarter of these diagnoses were minor according to the direct AIS coding.

\section{Abdominal or pelvic content}

The main problem of misclassifications by ICD-AIS map among abdominal injuries mainly concerned injuries of visceral organs. These injuries have mainly only one ICD-10 injury code per visceral organ in use in Finland regardless of whether the injury is a contusion

Table 5

ICD-AIS map severity rating (AIS 3+ or not) of injuries by ISS body region.

\begin{tabular}{|c|c|c|c|c|c|c|c|}
\hline $\begin{array}{l}\text { ICD-AIS map } \\
\text { rating result }\end{array}$ & Head and neck & Face & Chest & Abdominal or pelvic content & Extremities & External & Total \\
\hline Correctly classified & $252(75 \%)$ & $123(76 \%)$ & 337 (63\%) & $127(66 \%)$ & $454(82 \%)$ & $5(24 \%)$ & $1298(72.0 \%)$ \\
\hline Misclassified serious as minor & 25 & 2 & 111 & 49 & 29 & 0 & $216(12.0 \%)$ \\
\hline Misclassified minor as serious & 22 & 1 & 29 & 2 & 1 & 3 & $58(3.2 \%)$ \\
\hline Undetermined (minor injury) & 6 & 0 & 3 & 10 & 7 & 0 & $26(1.4 \%)$ \\
\hline Undetermined (serious injury) & 9 & 0 & 0 & 3 & 8 & 0 & $20(1.1 \%)$ \\
\hline Missing code & 20 & 36 & 59 & 1 & 55 & 13 & $184(10.2 \%)$ \\
\hline Total & 334 & 162 & 539 & 192 & 554 & 21 & $1802(100 \%)$ \\
\hline
\end{tabular}


Table 6A

Misclassified, missing, and undetermined ICD-10 codes in severity rating by ICD-AIS map.

\begin{tabular}{|c|c|c|c|}
\hline & Misclassified codes (n) & Missing codes $(n)$ & Undetermined codes $(\mathrm{n})$ \\
\hline \multirow[t]{6}{*}{ Head and neck } & S02.0 (7) & S01.7 (5) & S06.8 (12), S06.9 \\
\hline & S06.0, S06.2 (4), S06.3 (11) & S02.01, S02.71 (2), S02.7 (3), S06.7 & S07.9 \\
\hline & S06.4 (3), S06.6 (8) & S12.7 (5), S15.7 (2) & S19.8 \\
\hline & S12.0, S12.1 (2), S12.2 (3) & S16.0 & \\
\hline & S13.0, S14.3 & & \\
\hline & S15.0 (3), S15.1 (2) & & \\
\hline \multirow[t]{4}{*}{ Face } & S02.4 (2), S02.41 & S00.7 (4), S01.7 (8), & \\
\hline & & S02.30, S02.47 (3), S02.53, S02.54 & \\
\hline & & S02.7 (11), S02.70 (2), S02.71 (2) & \\
\hline & & S02.80, S03.21, S06.28 & \\
\hline \multirow[t]{6}{*}{ Chest } & S12.2 & S22.1 (57) & S27.6, S27.9 \\
\hline & S22.0 (4), S22.3 (6), S22.4 (91) & S26.8 (2) & S29.8 \\
\hline & S23.0 & & \\
\hline & S27.0 (7), S27.2 (2), S27.3 (26) & & \\
\hline & S29.0 & & \\
\hline & S32.0 & & \\
\hline \multirow[t]{4}{*}{ Abdominal or pelvic content } & S32.0 (3), S33.0, S34.2, S34.4, S35.5 & S36.7 & S35.8, S35.9 \\
\hline & S36.0 (17), S36.1 (13), S36.2, & & S36.8 (7) S36.9 \\
\hline & S36.4 & & S37.8 (3) \\
\hline & S37.0 (10), S37.2, S37.3 & & \\
\hline \multirow[t]{9}{*}{ Extremities } & S25.3 & S32.7 (26), S33.7 & S35.8, S44.8, S57.8 \\
\hline & S32.0, S32.1, S32.5 & S47.0 & S63.4, S66.1 \\
\hline & $S 42.3$ (2), S42.4 & S51.1, S51.7, S52.4 (4) & S77.1 (4) \\
\hline & S52.0, S52.2 (2), S52.5, S52.6 (2) & S60.7 (2), S61.7, S61.8 & S87.0, S87.8 (4) \\
\hline & S65.0 & S62.4 (3), S62.7 (3) & S89.9 \\
\hline & S82.1 (4), S82.2 (6), S82.3 (4), S82.4, S82.8 & S72.7 (2) & \\
\hline & & S82.7 & \\
\hline & & S92.7 (4), S93.2 (3) & \\
\hline & & T01.9 & \\
\hline \multirow[t]{2}{*}{ External } & T21.2, T21.3 & T00.9 (12), T01.9 & \\
\hline & $\mathrm{T} 24.3$ & & \\
\hline
\end{tabular}

Table 6B

Injury severity ratings by ICD-AIS map and by direct coding of the most common injury codes with errors.

\begin{tabular}{|c|c|c|}
\hline Injury codes with most common conversion errors (shown in bold in Table 6A) & Severity rating by ICD-AIS map & Severity rating by direct coding \\
\hline \multicolumn{3}{|l|}{ Head and neck } \\
\hline S06.3 & serious & $70 \%$ serious, $30 \%$ minor \\
\hline S06.6 & minor & $22 \%$ serious, $78 \%$ minor \\
\hline S02.0 & minor & $64 \%$ serious, $36 \%$ minor \\
\hline S0X.7, S1X.7, S0X.8, S1X.8, S0X.9, S1X.9 & missing or undetermined & $52 \%$ serious, $48 \%$ minor \\
\hline \multicolumn{3}{|l|}{ Face } \\
\hline Multiple missing codes (see Table 6A) & missing & $19 \%$ serious, $81 \%$ minor \\
\hline \multicolumn{3}{|l|}{ Chest } \\
\hline S22.4 & minor & $88 \%$ serious, $12 \%$ minor \\
\hline S27.3 & serious & $76 \%$ serious, $24 \%$ minor \\
\hline S22.1 & missing & $4 \%$ serious, $96 \%$ minor \\
\hline \multicolumn{3}{|l|}{ Abdominal or pelvic content } \\
\hline S36.X & minor, missing, or undetermined & $49 \%$ serious, $51 \%$ minor \\
\hline S37.X & minor or undetermined & $67 \%$ serious, $33 \%$ minor \\
\hline \multicolumn{3}{|l|}{ Extremities } \\
\hline Open fractures S42.X, S52.X, S62.X, S82.X, S92.X & $94 \%$ minor, $6 \%$ missing & $48 \%$ serious, $52 \%$ minor \\
\hline S32.7 & missing & $93 \%$ serious, $7 \%$ minor \\
\hline \multicolumn{3}{|l|}{ External } \\
\hline T00.9 & missing & minor \\
\hline
\end{tabular}

or a rupture or minor or major. According to AIS 2005/2008, major contusions and lacerations of visceral organs are as a rule considered serious injuries. In the ICD-AIS map these injuries are considered serious only by subcodes (ICD-10-CM) that are not used in Finland. Consequently, the codes used in Finland are systematically classified as minor by the ICD-AIS map. In our data, over half of visceral injuries were serious according to the direct AIS coding.

\section{Injuries of extremities}

There were multiple cases of missing codes in the ICD-AIS map among injuries of extremities; half of them were serious. Most of the missing cases were injury codes S32.7 "Multiple fractures of lumbar spine and pelvis" of which almost all were serious. The ICDAIS map underclassified injuries of extremities most commonly in open fractures of the humerus, forearm, wrist, hand, lower leg, ankle, and foot. In ICD-10 injury codes used in Finland there are no separate codes for open and closed fractures regarding extremities. However, certain open fractures are determined as serious according to AIS 2005/2008. The ICD-AIS map separates them with subcodes (ICD-10-CM), which are not in use in Finland. Due to the overly simple ICD-10 coding regarding fractures of the extremities, the ICD-AIS map systematically underclassified certain open fractures. 


\section{External injuries}

External injuries are a marginal group in our data in the context of injury severity. External injuries include lacerations, contusions, abrasions and burns, independent of their location on the body surface. In our data, none of these injuries was serious by direct AIS coding. However, the ICD-AIS map classified three injuries as serious. These injuries were $2^{\text {nd }}$ or $3^{\text {rd }}$ degree burns. Furthermore, there was one common injury code T00.9 "Multiple superficial injuries" (unspecified) that does not exist in the ICD-AIS map. In practice, these injuries were abrasions and contusions on different parts of the body.

\section{Comparison with previous studies}

When evaluating the reliability of the ICD-AIS map with respect to its main purpose in Finland, the number of MAIS 3+ patients is the most important information. In our HTR data, the ICD-AIS map underestimated the number of serious injuries (AIS $3+$ ) by $36 \%$ and the number of seriously injured patients (MAIS 3+) by $21 \%$. In the previous study from Finland [9], the underestimation of seriously injured patents (MAIS $3+$ ) by the ICD-AIS map was higher (34\%). The difference is explained mainly by different data. The data in the earlier study [9] was from a level-II trauma centre and contained quite a small proportion (10\%) of seriously injured (MAIS $3+$ ) patients. On the other hand, in the present data all patients were seriously injured (MAIS $3+$ and NISS $>15$ ). Moreover, in the present data, the number of serious injuries per patient also was higher, and the possibility that even one serious injury code of a patient converted correctly was greater than in the earlier study. Furthermore, there are similarities in the results of the present and the previous study [9] when looking at the MAIS 3+ conversion results by injury mechanism. In both studies the proportions of correct results were highest in pedestrian accidents and lowest in motorcycle (and moped) accidents.

Similar results to our study have also been presented earlier by Pérez et al. [6] who compared the number of MAIS 3+ patients between the ICD-AIS map by AAAM and direct AIS coding. They used a German data set $(n=209)$ and concluded that the ICD-AIS map underestimated the amount of seriously injured patients by $20 \%$ [6], which is nearly the same figure as ours. However, they used a German version of ICD-10 (ICD-10-GM) which is, to some extent, more specific than the ICD-10 used in Finland. In general, several studies indicate that different kinds of automatic ICD-AIS conversion tools generates rather lower than higher numbers of serious injuries than direct AIS coding [6,14-16]. The reliability of input data, and more specifically, the quality and accuracy of ICD injury coding, is essential for the successful conversion and has a significant impact on the results. Pérez et al. [6] have made recommendations on how to address the challenges of inadequate data.

It is not reasonable to compare all previous findings regarding various conversion tools to ours. In addition to different tools, there is considerable variation in the used ICD classifications and AIS versions. Glerum and Zonfrillo [15] have comparable results closest to ours. They compared the agreement of AIS scores between ICD-AIS map by AAAM and direct AIS-coding by trained coders. They used data from the Harborview Medical Center (Seattle, Washington, USA) trauma registry, including a total of 1990 trauma patients with ICD-10-CM injury codes. Furthermore, the AIS rating results were compared according to ISS body regions. The highest agreements of AIS scores between the ICD-AIS map and direct AIS coding were among injuries of extremities (84\%) and external injuries (82\%) and lowest among head and neck injuries (44\%) [15]. The results differed from ours especially in external injuries and head and neck injuries. Presumably the main reason for the different results was the use of ICD-10-CM injury codes; we used the more simple ICD-10. Furthermore, Glerum and Zonfrillo [15] used all AIS classes [16], whereas we used only two (AIS 1-2 and AIS 3+). However, they highlighted some of the same challenges as we did regarding determination of the severity of specific head injuries.

\section{The significance of ICD-10 coding}

In Finland, the number of seriously injured patients is determined based on the injury codes from the Finnish Hospital Discharge Registry (FHDR). Heinänen et al. [11] evaluated the coverage and accuracy of both the FHDR and the HTR (previously TR-THEL) by comparing them with the original patient files and trauma registry files from the trauma registry of the Helsinki University Hospital Trauma Unit. The coverage and accuracy of diagnoses in the Finnish Hospital Discharge Register were 65.5\% (95\% CI: 62.5\%-68.5\%) and 73.8\% (95\% CI: 70.4\%-77.2\%), respectively. Of all the missing diagnosis of FHDR (333/965), 23\% were serious (AIS 3+) [11]. The coverage and accuracy of diagnosis of HTR were evaluated as excellent with figures of 95.8\%(95\% CI: 94.5\%-97.0\%) and 97.6\% (95\% CI: 96.7\%-98.6\%), respectively. This indicates that the errors in injury coding did not cause uncertainty in the results of the present study. However, at the national level, when using the data of FHDR for determining the number of seriously injured by ICD-AIS map, the lack of coverage and accuracy of injury coding probably causes some errors. Most likely, the underestimation of MAIS 3+ patients at the national level is actually even greater than the present study and that of previous study.

According to the earlier study by Airaksinen et al. [9], the main reason for the misclassifications by the ICD-AIS map was the simplicity of the ICD-10 classification used in Finland compared to ICD-10-CM used in the ICD-AIS map. In Finland, only four- or five-digit injury codes are generally in use. In the ICD-AIS map, there is ca. 16,500 injury codes whereas in the version used in Finland, the number of codes is ca.1,400. The same problem emerged in the present study. Pérez et al. [6] have also concluded that because most European countries use an older version of ICD-10 without clinical modification (CM), the conversion table does not fit well with European practice. As in the previous study, misclassifications appeared frequently in the injuries of visceral organs such as major ruptures, in open fractures of extremities, and in specific head injuries with prolonged unconsciousness. A new finding in the present study is the high number of cases with more than two fractured ribs, which is a serious injury and problematic for the conversion. Neither the ICD-10 code of the injury (S22.4) used in Finland nor the ICD-AIS map separates the number of fractured ribs. This led to systematic errors. In addition, in the present study, we obtained plenty of new important information on numerous undefined and missing codes of the ICD-AIS map and their prevalence in Finnish HTR data. It is noteworthy that except for the previous Finnish study [9], there are no other reference studies with equally detailed conversion analysis.

The World Health Organization released a new International Classification of Diseases (ICD-11) in June 2018 [17]. ICD-11 provides significant improvements compared to present ICD-10 versions. The injury codes in ICD-11 are more detailed than those in ICD-10. However, although the ICD-11 complete, it will not be deployed in the near future. The WHO has planned for its deployment in 2022. Meanwhile, the member countries are planning how to use the new version, preparing translations, and training health professionals [18]. In terms of the severity rating of injuries, it would be very important to introduce all detailed injury codes of ICD-11.

\section{Strengths and limitations}

The strength of our study is the reliability of the HTR data. The coverage and accuracy of the injury coding has been evaluated as 
excellent [11]. Therefore, the conversion results do not include uncertainty due to data. However, the data include only the most seriously injured victims (NISS $>15$ ) of road traffic accidents in the catchment area of 1.8 million inhabitants and thus do not represent all road traffic accident patients in Finland. As the HTR contains more comprehensive data, the reliability of ICD-AIS map in identifying seriously injured patients may be preferable to the national FHDR data.

\section{Conclusions}

The number of seriously injured patients (MAIS 3+) was one fifth lower and the number of serious injuries (AIS 3+) was more than a third lower by ICD-AIS map than by direct coding. The main reason for misclassifications by the ICD-AIS map was the simplicity of the ICD-10 codes used in Finland compared to those of the ICD$10-\mathrm{CM}$ used in the ICD-AIS map. The most frequent errors were due to multiple fractures of ribs, injuries of visceral organs, some open fractures of extremities, and specific head injuries with prolonged unconsciousness. The ICD-10 injury codes presently used in Finland should be more specific to permit reliable conversion results by the ICD-AIS map. Furthermore, the problem with missing codes should be considered more closely: is it a deficiency of the ICD-AIS map or due to updates or reclassifications of ICD-10 (used in Finland) or ICD-10-CM (used in map) that might cause different coding of certain injuries. Moreover, when implementing the ICD-11, all detailed injury codes should be introduced.

\section{Declaration of Competing Interest}

The authors declare that there are no financial or personal relationships with other people or organizations that could cause conflicts of interest.

\section{Acknowledgements}

The authors received no financial support for the research of this article. The authors had no writing assistance.

\section{References}

[1] High Level Group on Road Safety consultation on the development of the injuries strategy. 2nd Working Document: next steps in the development of the injuries strategy final. 2012. . November, Available at: https://ec.europa.eu/ transport/road_safety/sites/roadsafety/files/pdf/ser_inj/ser_inj_breen.pdf.
[2] Association for the Advancement of Automotive Medicine (AAAM). The abbreviated injury scale (AIS) 2005 - update 2008.. Barrington, IL: AAAM; 2008.

[3] Zonfrillo MR, Weaver AA, Gillich PJ, Price JP, Stitzel JD. New methodology for an expert-designed map from International Classification of Diseases (ICD) to Abbreviated Injury Scale (AIS) 3+ severity injury. Traffic Inj Prev 2015;16 (Suppl. 2):197-200, doi:http://dx.doi.org/10.1080/15389588.2015.1054987.

[4] Loftis KL, Price JP, Gillich PJ, Cookman KJ, Brammer AL, St Germain T, et al. Development of an expert based ICD-9-CM and ICD-10-CM map to AIS 2005 update 2008. Traffic Inj Prev 2016;17(Suppl. 1):1-5, doi:http://dx.doi.org/ 10.1080/15389588.2016.1191069.

[5] Pérez K, Weijermars W, Amoros E, Bauer R, Bos N, Dupont E, et al. Practical guidelines for the registration and monitoring of serious traffic injuries, D7.1 of the H2020 project SafetyCube.2016. Available at:. https://dspace.lboro.ac.uk/ dspace-jspui/bitstream/2134/23760/1/ SC_D7.1_Guidelines_Serious_Injuries_finalV2.pdf.

[6] Pérez K, Weijermarsd N, Bosd A, Filtnesse R, Bauerf H, Johannseng N, et al. Implications of estimating road traffic serious injuries from hospital data. Accid Anal Prev 2018, doi:http://dx.doi.org/10.1016/j.aap.2018.04.005 [Epub ahead of print].

[7] Baker SP, O'Neill B, Haddon Jr W, Long WB. The injury severity score: a method for describing patients with multiple injuries and evaluating emergency care. J Trauma 1974;14(3):187-9.

[8] Osler T, Baker SP, Long W. A modification of the Injury Severity Score that both improves accuracy and simplifies scoring. J Trauma 1997:43:922-5.

[9] Airaksinen N, Nurmi-Lüthje I, Kröger H, Lüthje P. The ability of the ICD-AIS map to identify seriously injured patients in road traffic accidents - a study from Finland. Traffic Inj Prev 2018;19(8):819-24, doi:http://dx.doi.org/10.1080/ 15389588.2018 .1520985$.

[10] Eid HO, Abu-Zidan FM. New Injury Severity Score is a better predictor of mortality for blunt trauma patients than the Injury Severity Score. World J Surg 2015;39:165-71, doi:http://dx.doi.org/10.1007/s00268-014-2745-2.

[11] Heinänen M, Brinck T, Handolin L, Mattila VM, Söderlund T. Accuracy and coverage of coding of severely injured patients in FHDR and TR-THEL. Scand J Surg 2017;106(3):269-77, doi:http://dx.doi.org/10.1177/1457496916685236.

[12] Heinänen M, Brinck T, Leferin R, Handolin L, Söderlund T. How to validate the data quality in a Trauma Registry? -Helsinki Trauma Registry internal audit. 2019 Submitted.

[13] Association for the Advancement of Automotive Medicine (AAAM): "Copy of aaam_icd10map_v1 0_Feb2015 read only.xls". 2015.

[14] Fleischman RJ, Mann NC, Dai M, Holmes JF, Wang NE, Haukoos J, et al. Validating the use of ICD-9 code mapping to generate injury severity scores. Trauma Nurs 2017;24(1):4-14, doi:http://dx.doi.org/10.1097/ JTN.0000000000000255.

[15] Glerum KM, Zonfrillo MR. Validation of an ICD-9-CM and ICD-10-CM map to AIS 2005 Update 2008. Inj Prev 2019;25:90-2, doi:http://dx.doi.org/10.1136/ injuryprev-2017-042519.

[16] Haas B, Xiong W, Brennan-Barnes M, Gomez D, Nathens AB. Overcoming barriers to population-based injury research: development and validation of an ICD-10-to-AIS algorithm. Can J Surg 2012;55:21-6, doi:http://dx.doi.org/ $10.1503 /$ cjs.017510.

[17] The World Health Organization (WHO). WHO releases new International Classification of Diseases (ICD 11), press release. 18th June 2018, Geneva. https://www.who.int/news-room/detail/18-06-2018-who-releases-newinternational-classification-of-diseases-(icd-11).

[18] The World Health Organization (WHO). ICD-11 International Classification of Diseases 11th Revision. The global standard for diagnostic health information. 2019. https://icd.who.int/. 